\title{
ON THE TOPOLOGY OF THE SET OF COMPLETELY UNSTABLE FLOWS
}

BY

\author{
ZBIGNIEW NITECKI
}

\begin{abstract}
We show that: (1) on any open manifold other than the line or plane, there exist nonsingular flows with $\Omega \neq \varnothing$ which can be perturbed, in the strong $C^{r}$ topology (any $r$ ), to flows with $\Omega=\varnothing$, and that (2) on certain open 3-manifolds there exist flows with $\Omega=\varnothing$ which cannot be approximated, in the strong $\mathcal{C}^{1}$ topology, by flows satisfying both $\Omega=\varnothing$ and no $\mathcal{C}^{1}$ $\Omega$-explosions. These examples give partial negative answers to the conjecture of Takens and White, that the completely unstable flows with the strong $\mathcal{C}$ topology equal the closure of their interior.
\end{abstract}

Takens and White [10] have conjectured that the collection of completely unstable flows on an open manifold is the closure of its interior. This paper presents several counterexamples to their conjecture.

We recall some definitions. Let $\mathbf{M}$ be an open (finite-dimensional, paracompact, noncompact, connected, boundaryless) manifold; let $\mathfrak{X}^{r}(\mathbf{M})$ denote the collection of $e^{r}$ vectorfields on $M$ which generate flows; we shall look at an element of $\mathfrak{X}^{r}$ as either a vectorfield or the flow it generates. $\mathfrak{X}^{r}$ is given the strong $C$ topology: a neighborhood of a vectorfield is determined by a positive continuous function $\varepsilon(x)$ on $\mathrm{M}$, which replaces the constant $\varepsilon$ in the pointwise estimates defining the usual $C^{r}$ topology (for compact manifolds). We consider three subsets of $\mathfrak{X}^{r}(\mathbf{M}): \mathfrak{N}(\mathbf{M})$ denotes the set of nonvanishing vectorfields on $\mathbf{M}, \mathfrak{B r}^{r}$ denotes the set of completely unstable flows on $\mathbf{M}$ (that is, all orbits wander), and $\mathbb{S}^{r}(\mathrm{M})$ denotes the interior of $\mathfrak{W}^{r}$-the nonexplosive flows. Clearly, $\mathbb{S}^{r} \subset \mathfrak{B} \subset \mathfrak{M}$. The collection $\mathbb{S}^{\circ}(\mathrm{M})$ has been characterized [4] as the set of gradient flows for functions without critical points.

As stated, the conjecture of Takens and White is that $\mathfrak{B r}(\mathrm{M})$ is the closure of $\mathrm{CS}^{\mathrm{r}}(\mathrm{M})$ for all $r \geqslant 1$ and all open manifolds $\mathrm{M}$. By slowing down a vectorfield (in $\mathfrak{B}$ ) to zero at one point, it is easy to see that $\mathfrak{B}$ is not closed in $\mathfrak{R}^{r}$. However, the spirit of the conjecture refers really to the foliation by orbits of the flow, and so the content of the conjecture is the following pair of statements:

(a) $\mathfrak{B}^{r}(\mathbf{M})$ is closed in $\mathfrak{R}^{r}(\mathbf{M})$,

(b) $\mathfrak{S}^{r}(\mathbf{M})$ is dense in $\mathfrak{B}^{r}(\mathbf{M})$.

Received by the editors April 26, 1978.

AMS (MOS) subject classifications (1970). Primary 58F10; Secondary 34D10.

Key words and phrases. Completely unstable flow, $\Omega$-explosion, plug, strong topology.

(C) 1979 American Mathematical Society 0002-9947/79/0000-0356/\$05.00 
In particular, when $\mathbf{M}=\mathbf{R}^{1}$ or $\mathbf{R}^{2}$, then by the Poincare-Bendixson theorem (s) $^{r}(\mathrm{M})=\mathfrak{B}^{r}(\mathrm{M})=\mathfrak{N}^{r}(\mathrm{M})$ for all $r$, and both statements above are true.

On the other hand, it is known [5] that for any open manifold $M$ other than the line or plane, the containments $\mathbb{S} \subset \mathfrak{W} \subset \mathfrak{N}$ are both proper. The counterexamples in this paper establish the following negations of the statements above:

THEOREM 1 For any open manifold $\mathrm{M}$ not homeomorphic to $\mathrm{R}^{1}$ or $\mathrm{R}^{2}$, and any $r \geqslant 0, \mathfrak{W}^{r}(\mathrm{M})$ is not closed in $\mathfrak{N}(\mathbf{M})$.

THEOREM 2. Let $\mathrm{X}$ by any 3-manifold (open or closed) and let $\mathrm{K}$ be either the union of two unlinked, unknotted contractible circles, or the simply-embedded figure-eight obtained by joining two such circles at a point. Let $\mathrm{M}$ denote the open manifold $\mathrm{X}-\mathrm{K}$. Then for $r \geqslant 1$, $\mathbb{S}^{r}(\mathrm{M})$ is not dense in $\mathfrak{F}^{r}(\mathrm{M})$.

The examples proving Theorem 1 are types of saddle-node bifurcations, while Theorem 2 is proven by a slight variation of the example of Pugh, Walker, and Wilson [9]. I would like to particularly thank Alan Dankner for directing my attention to [9], and to acknowledge the partial support of the great blizzard of February 6-7, 1978-which, by closing New England for a week, provided released time during which this paper was written.

1. TheOREM 1. $\mathfrak{B}$ is not closed.

Let $\mathbf{M}$ be an open manifold not homeomorphic to either the line or plane. We shall show that $\mathfrak{W}^{r}(\mathrm{M})$ is not closed in $\mathscr{N}(\mathrm{M})$ by producing a continuous arc $\mathfrak{X}:[0,1] \rightarrow \mathfrak{N}(M)$ such that $\mathscr{X}_{u} \in \mathfrak{W}$ for $u<1$ but $\Omega\left(\mathscr{X}_{1}\right) \neq \varnothing$. For most manifolds, our construction will be based on:

LEMMA 1. Let $\mathscr{X}_{0}$ denote the vectorfield on the cylinder $[-1,1] \times S^{1}$ defined by $\mathfrak{X}_{0}(r, \theta)=(1,0)$. There exists an arc of vectorfields $\mathfrak{X}_{u}, 0 \leqslant u \leqslant 1$, on $[-1,1] \times S^{1}$ such that:

(i) $\mathscr{X}_{u}=\mathscr{X}_{0}$ on $\{ \pm 1\} \times S^{1}$ for all $u$,

(ii) for every $u<1$, and any $\theta \in S^{1}$, the points $(-1, \theta)$ and $(+1, \theta)$ lie on the same integral curve of $\mathfrak{X}_{u}$,

(iii) $\mathfrak{X}_{1}$ has two periodic orbits.

Proof. Let $f(r)$ be a $\bigodot^{\infty}$ function satisfying:

(a) $f(-r)=-f(r)$,

(b) $f(r)=0$ for $|r|>2 / 3$,

(c) $f(1 / 3)=\pi / 2$ is a strict global maximum.

Then define $\mathscr{X}_{u}$ by

$$
\mathcal{X}_{u}(r, \theta)=(\cos [u f(r)], \sin [u f(r)]) .
$$

(See Figure 1.) Statements (i) and (iii) are clearly true; (ii) follows from (a) 
and the fact that if $u<1, \mathfrak{X}_{u}$ has a nonvanishing component in the $r$ direction. Q.E.D.

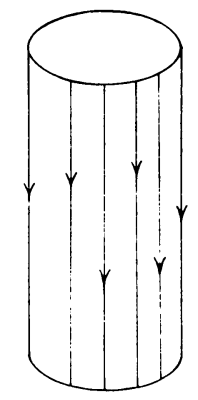

$u=0$

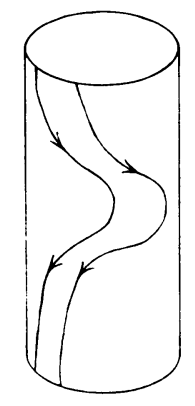

$0<u<1$

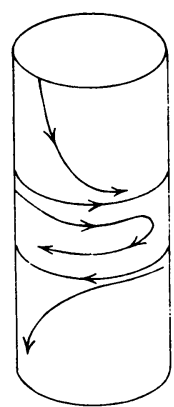

$u=1$

FIGURE $1 \quad x_{u}$

For higher dimensions, we extend Lemma 1 to

Corollary 1. Let $\mathrm{A}=[-1,1] \times \mathrm{S}^{1} \times \mathrm{D}^{k}$, where $\mathrm{D}^{k}$ denotes a $k$-disc $(k>1)$. Then there exists an arc of vectorfields $\mathfrak{X}_{u}, 0<u<1$, on A such that

(i) $\mathfrak{X}_{u}(r, \theta, \vec{x})=(1,0, \overrightarrow{0})$ on $\partial \mathrm{A}$ for all $u, 0<u<1$, and on all of $\mathrm{A}$ for $u=0$,

(ii) for every $u<1,(+1, \theta, \vec{x})$ and $(-1, \theta, \vec{x})$ lie on the same integral curve of $\mathfrak{X}_{u}$,

(iii) $\mathfrak{X}_{1}$ has two periodic orbits.

Proof. Take a $\bigodot^{\infty}$ function $g: D^{k} \rightarrow[0,1]$ with support interior to $D^{k}$ and $g^{-1}(1)=\{\overrightarrow{0}\}$. Then $\mathscr{X}_{u}(r, \theta, \vec{x})=(\cos [u f(r) g(\vec{x})], \sin [u f(r) g(\vec{x})])$ has the required properties. Q.E.D.

We use the corollary to prove Theorem 1 on any open manifold of dimension 3 or more:

Proposition 1. If $\mathrm{M}$ is open and $\operatorname{dim} m=n>3$, then $\mathfrak{B r}^{r}(\mathrm{M})$ is not closed in $\mathfrak{N}^{r}(\mathrm{M})$.

Proof. Using [1], we take a $\bigodot^{\infty}$ function $F: M \rightarrow R$ without critical points, and let $\mathcal{X}$ be its gradient (relative to some Riemann metric). Then $\mathcal{X} \in$ $\mathfrak{B}^{r}(\mathrm{M})$. Pick a point $x_{0} \in \mathrm{M}$, and take a disc $\mathrm{T} \subset \mathrm{M}$ through $x_{0}$ transverse to $\mathcal{X}$. Let $\mathrm{C}$ be a circle in $\mathrm{T}$, and thicken $\mathrm{C}$ to a diffeomorphic image of $S^{1} \times D^{n-2}$. Finally, thicken this to a set homeomorphic to $A$ (of the corollary), using the flow. Inside $\mathrm{A}, \mathfrak{X}$ is conjugate to $\mathscr{X}_{0}$ of Corollary 1 , so that the arc $\mathfrak{X}_{u}$ can be extended to all of $M$ by (i). That $\mathscr{X}_{u} \in W^{r}(M)$ for $u<1$ follows from (ii). Since $\mathfrak{X}_{u}$ has compact support, it is continuous as an arc in $\mathfrak{X}^{r}(\mathbf{M})$ by [6]. Thus, $\mathfrak{X}_{1}$ belongs to clos[Wr(M)] - $\mathfrak{F}^{r}(\mathbf{M})$. Q.E.D. 
From the argument above, it is clear that Lemma 1 can be used to prove Theorem 1 on any surface $M$ in which we can find a flow $\phi \in \mathfrak{B}(M)$ and a circle $\mathbf{C} \subset \mathbf{M}$ transverse to $\mathcal{X}=\dot{\phi}$.

LEMMA 2. If $\mathrm{M}$ is an open orientable surface containing a nonbounding circle $\mathrm{C}$, there exists a flow $\phi \in \mathfrak{B}^{r}(\mathrm{M})$ with $\dot{\phi}$ transverse to $\mathrm{C}$.

Proof. Define a $e^{\infty}$ function $f_{1}$ on a tubular neighborhood $\mathrm{A}$ of $\mathrm{C}$ (an annulus), so that $\mathrm{C}$ is a level set and $f_{1}$ has no critical points on $\mathrm{A}$. By successive application of Tietze's theorem, the density of smooth functions [2, p. 44], and the density of Morse functions [2, p. 147], we obtain a $e^{\infty}$ extension $f_{2}$ of $f_{1}$, which agrees with $f_{1}$ near $\mathrm{C}$ and has only isolated critical points, none near C. The critical points of $f_{2}$ can be joined to "infinity" by a countable family of disjoint arcs which are closed embeddings of $[0,1)$ in $M$ and, since $C$ is nonbounding, they can be chosen to miss $C$. But the complement of these arcs in $\mathbf{M}, \overline{\mathbf{M}}$, is diffeomorphic to $\mathbf{M}$ itself, and $\operatorname{grad} f_{2} \in \mathfrak{W}^{\prime}(\overline{\mathbf{M}})$ is transverse to C. Q.E.D.

PROPOSITION 2. If $\mathbf{M}$ is an orientable open surface other than $\mathbf{R}^{2}$, then $\mathfrak{B}^{r}(\mathrm{M})$ is not open in $\mathfrak{N}(\mathrm{M})$.

PROOF. This is a corollary of Lemmas 1 and 2. Q.E.D.

We could actually have allowed $M$ in Lemma 2 and Proposition 2 to be any (possibly nonorientable) surface which has a handle; this would exclude only the punctured projective plane and punctured Klein bottle. However, the argument which works for these surfaces can be adapted as well to any open nonorientable surface. Using the surgery technique of [5], the problem boils down to the case of punctured closed surfaces:

PROPOsITION 3. If $\mathrm{X}$ is a closed nonorientable surface and $\mathrm{K}$ is a point, then there exists an arc $\mathfrak{X}:[0,1] \rightarrow \mathfrak{N}^{(}(\mathrm{X}-\mathrm{K})$ such that $\mathfrak{X}_{u} \in \mathfrak{W}^{r}(\mathbf{X}-\mathbf{K})$ for $u<1$, but $\Omega\left(\mathscr{X}_{1}\right) \neq \varnothing$.

Proof. Represent $\mathrm{X}$ by a polygon with pairwise identification of the edges, and all vertices corresponding to $K$. As in [5, §3], define an initial flow by filling the interior of the polygon with loops from a single vertex.

We claim, if $\mathrm{X}$ is nonorientable, that at least one pair of edges can be made into an orbit of the flow-that is, the identification respects the orientation induced on these edges by the flow. To see this (Figure 2), take an arc $\gamma$ joining an identified pair of points on two vertices, and consider an orthonormal frame along $\gamma$ whose first element is $\dot{\gamma}$ and whose second element is initially tangent to the first edge, in the direction of the flow. At the end of $\gamma$, the second element is opposed to the flow; if this is consistent with the identification, then orientation does not change in $\mathrm{X}$ as one crosses the cycle corresponding to this edge. But $\mathrm{X}$ is nonorientable, so orientation 


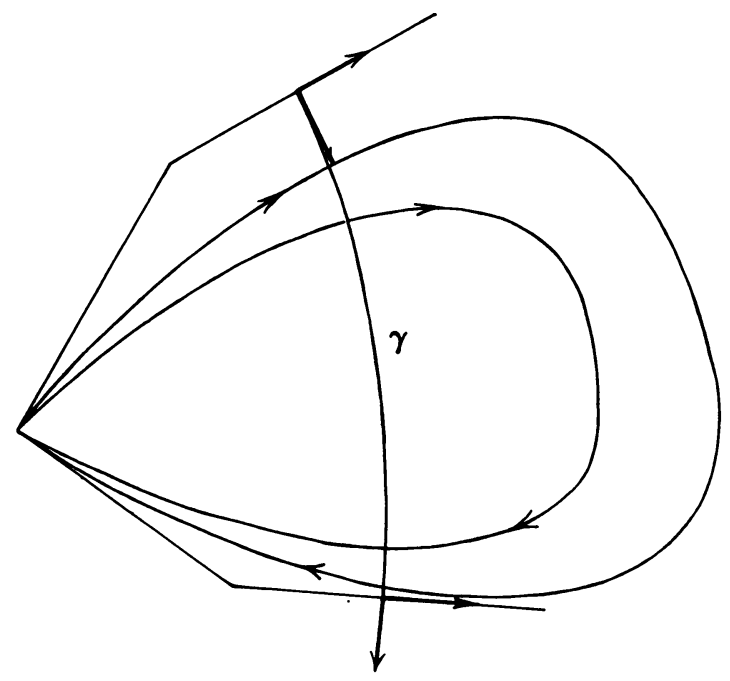

FIGURE 2

must reverse across some edge-cycle; this cycle can be made an orbit of the flow.

For all other cycles, we introduce either a parallel band or a Reeb component to separate the two sides of the cycle in the flow. Note that the one cycle which is a single orbit is nonwandering for the flow; this flow will be $\mathcal{X}_{1}$ in our proposition.

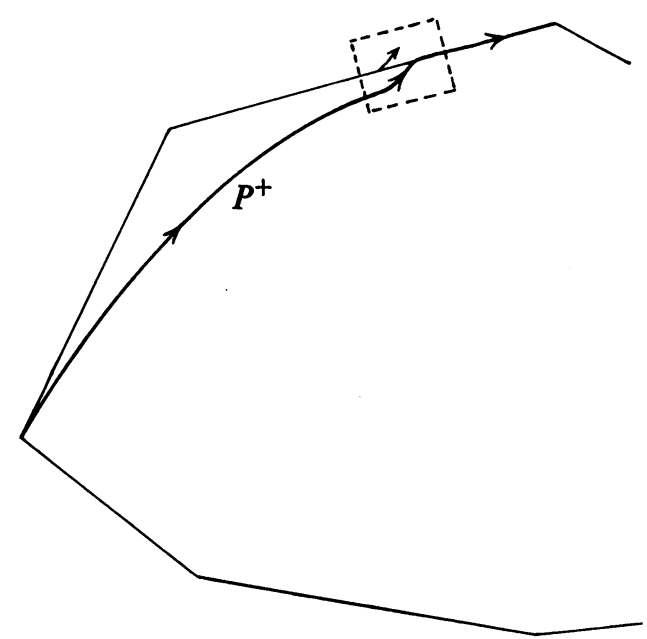

FigURE 3a 


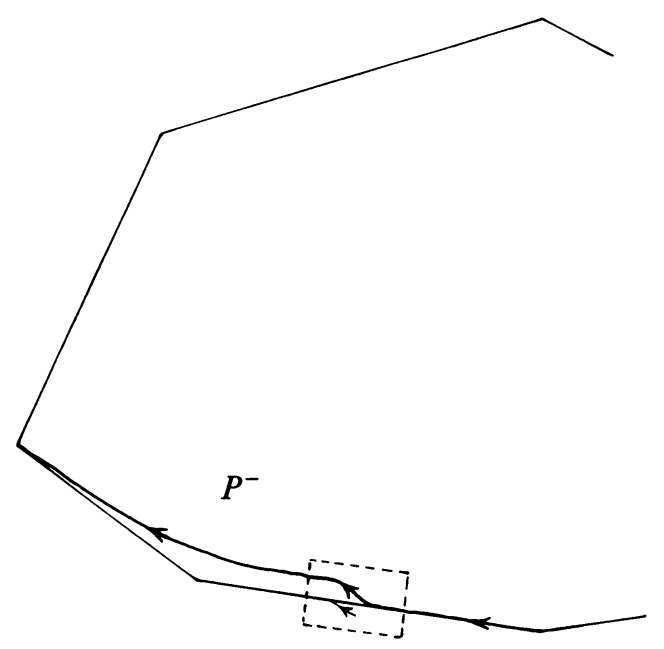

FIGURE 3b

On the other hand, take a small flowbox at a point on this cycle, and consider any perturbation which pushes the vectorfield out of the polygon on the edge which occurs earlier in the flow (and hence into the polygon on the later edge). The cycle now breaks into two orbits: $P^{+}$, whose forward trajectory coincides with part of the cycle, but whose backward trajectory agrees outside the box with the initial part of a loop (Figure $3 a$ ), and $P_{-}$, whose backward trajectory coincides with part of the cycle and whose forward trajectory follows the last part of a loop back to the initial vertex (Figure 3b).

We claim this perturbed flow is completely unstable in $X-K$. To see this, form two closed curves in $\mathrm{X}$, by (i) following $P^{+}$, then the edges of the polygon back to the starting point, and (ii) following edges and then $P^{-}$back to the starting point. These curves bound the saturation in $\mathrm{X}$ of the segment of the cycle between its junctures with $P^{+}$and $P^{-}$; by making the flow transverse here, we insure that every point interior to this region wanders. On the other hand, a neighborhood of a point on $\boldsymbol{P}^{+}$or $\boldsymbol{P}^{-}$has one half that stays interior to the polygon and another half that stays in the region described above, coming near the other edge $\left(P^{-}\right.$or $\left.P^{+}\right)$but never near the first special orbit.

The flow initially described belongs to the closure of these completely unstable perturbations, but itself has nonwandering orbits. In fact, it is easy to perform the above perturbation with a one-parameter family of vectorfields to prove Proposition 3 as stated. Q.E.D.

THEOREM 1. On any open manifold $\mathrm{M}$ other than $\mathrm{R}^{1}$ or $\mathrm{R}^{2}, \mathfrak{W}^{r}(\mathrm{M})$ is neither closed nor open in $\mathfrak{R}(\mathrm{M})$. 
Proof. That $\mathfrak{W r}$ is not open is the main theorem of [5]. Propositions 1, 2, and 3 show that $\mathfrak{W}^{r}$ is not closed in $\mathfrak{N}(\mathrm{M})$ for all $\mathrm{M}$ required by the theorem, except nonorientable surfaces of the form $X-K$ (K a totally disconnected set with more than one point), or nonorientable surfaces of infinite genus. Proposition 3 easily adapts to the first exception (put the rest of $\mathrm{K}$ on a single loop far from the edge). To handle the second case, we invoke the procedures of [5, $\S 3$ and 4]. The manifold $M$ can be decomposed into $M=M_{0} \cup C_{1}$ $\cup \ldots \cup \mathrm{C}_{n}$, where $\mathrm{M}_{0}=\mathrm{X}-\mathrm{K}, \mathrm{K}$ finite, and each $\mathrm{C}_{i}$ is a distinct noncompact component of $\mathbf{M}-\mathbf{M}_{0}$. The technique of [5, Lemma 4] can be applied to our example in Proposition 3 above, yielding a flow on $\mathbf{M}_{\mathbf{0}}$ which is transverse to $\mathrm{M}_{0} \cap \mathrm{C}_{i}$ for $i>0$; then the technique of $[5,84]$ can be used to glue this example into a completely unstable flow on $\mathbf{M}$, by taking all the tangencies on $\mathbf{M}_{0} \cap \mathbf{C}_{0}$ into Reeb components inside thickened orbits of the original flow. Again, the argument of Proposition 3 gives us a continuous arc, with support in $M_{0}$, such that $\mathscr{X}_{u} \in \mathfrak{W}^{r}(M)$ for $u<1$ and $\Omega\left(\mathscr{X}_{1}\right) \neq \varnothing$. Q.E.D.

2. THEOREM 2. $\mathbb{S}$ is not dense in $\mathfrak{B}$.

We turn now to the more subtle part of the conjecture: that every completely unstable flow can be approximated by a nonexplosive flow. Using earlier results of Takens, it was shown in [10] that every flow can be $e^{r}$-approximated by a flow with no (er) $\Omega$-explosions. If the perturbed flow is completely unstable, it belongs to $\mathbb{S S}^{r}$; however, it is unclear from the proof of the approximation theorem whether the nonexplosive perturbation of a completely unstable flow can itself be chosen to be completely unstable. In this section, we give a class of examples on certain 3-manifolds for which such a choice is impossible, in the $\mathcal{C}^{\mathrm{l}}$ topology.

Pugh, Walker and Wilson [9] have constructed a nonsingular flow on $\mathrm{S}^{3}$ for which $\Omega$ consists of nine hyperbolic periodic orbits, but such that any Kupka-Smale $e^{l}$ perturbation has infinite $\Omega$. By deleting the nine circles in the original $\Omega$ from $S^{3}$, one immediately obtains a counterexample to the second assertion in the Takens-White conjecture, for $r \geqslant 1$ and $M=S^{3}-K$ ( $\mathrm{K}=$ nine circles). We shall use the special features of the strong topology to simplify the topology of the deleted set $\mathrm{K}$, and note that, using some observations in [8], $\mathrm{S}^{3}$ can be replaced in the construction by any 3-manifold.

Constructing plugs. The Pugh-Walker-Wilson example is based on the "plug" construction of Wilson. We recall the main features of this device. Suppose $\phi$ is a nonsingular flow and $P$ is a flowbox: $P$ is homeomorphic to $\mathrm{D}^{2} \times[-1,1]$; its boundary consists of a cylinder $S^{1} \times[-1,1]$ of orbit segments for $\phi$, and two discs, the inset $\partial^{-} P=D^{2} \times\{-1\}$ and the outset $\partial^{+} P=D^{2} \times\{+1\}$ (see Figure 4). We modify the flow inside $P$, introducing 


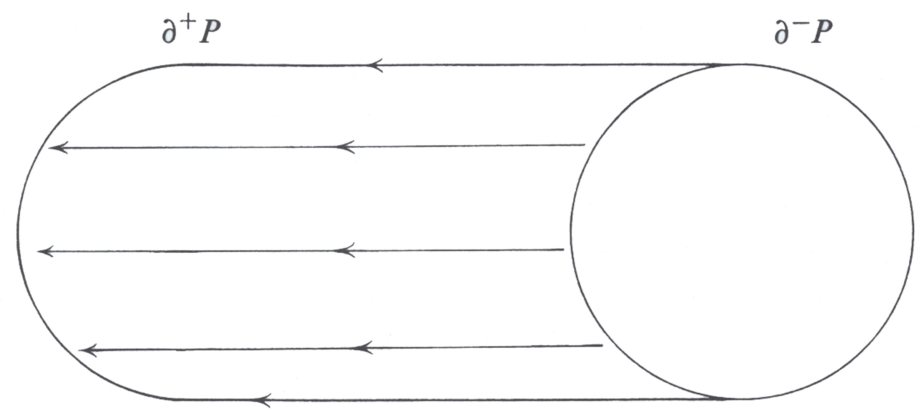

FIGURE 4

periodic orbits: the circles $\sigma_{-}=\mathrm{S}(1 / 2) \times\{-1 / 2\}$ and $\sigma_{+}=\mathrm{S}(1 / 2) \times$ $\{+1 / 2\}$ ( $\mathrm{S}(r)$ denotes a circle of radius $r$ ) are of saddle type, rotate in opposite directions, and are joined by a cylinder of saddle connections $\mathrm{W}^{s}\left(\sigma_{+}\right) \cap \mathrm{W}^{u}\left(\sigma_{-}\right)=\mathrm{S}(1 / 2) \times[-1 / 2,1 / 2]$. The other component of $\mathrm{W}^{s}\left(\sigma_{+}\right)\left(\right.$resp. $\left.\mathrm{W}^{u}\left(\sigma_{-}\right)\right)$forms a cylinder $\mathrm{S}(1 / 2) \times[1 / 2,3 / 4](\mathrm{S}(1 / 2) \times$ $[-3 / 4,-1 / 2])$ whose other boundary is a periodic source, $S(1 / 2) \times\{3 / 4\}$ (resp. sink, $S(1 / 2) \times\{-3 / 4\})$. The other invariant manifold, $\mathbf{W}^{u}\left(\sigma_{+}\right)$(resp. $\mathrm{W}^{s}\left(\sigma_{-}\right)$) forms two cone-like sheets meeting $\partial^{+} \mathrm{P}$ (resp. $\left.\partial^{-} \mathrm{P}\right)$ in the circles $\mathrm{C}_{o}^{+}=\mathrm{S}(3 / 4) \times\{+1\}, \mathrm{C}_{i}^{+}=\mathrm{S}(1 / 4) \times\{+1\} \quad\left(\right.$ resp. $\mathrm{C}_{o}^{-}=\mathrm{S}(3 / 4) \times\{-1\}$, $\left.\mathrm{C}_{i}^{-}=\mathrm{S}(1 / 4) \times\{-1\}\right)($ see Figure 5).

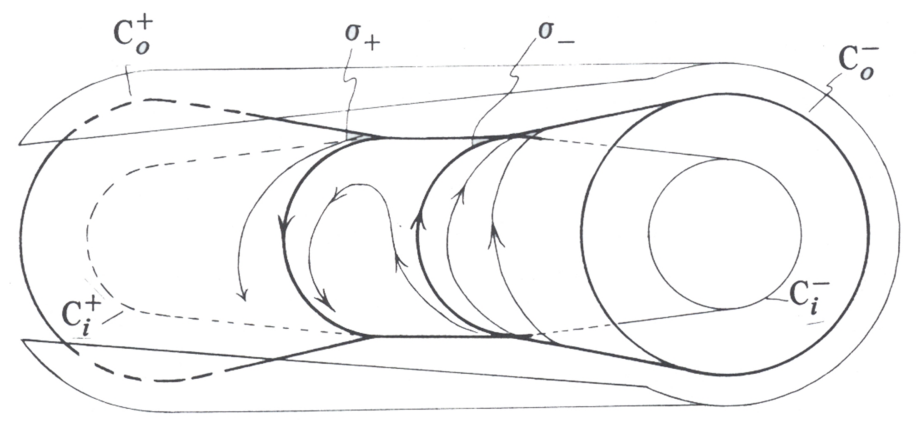

FIGURE 5

Note that an orbit entering the annulus $\mathrm{A}^{-}$, bounded by $\mathrm{C}_{0}^{-}$and $\mathrm{C}_{i}^{-}$, tends asymptotically to the closed sink; the corresponding statement holds for backward orbits crossing $\mathrm{A}^{+}$(bounded by $\mathrm{C}_{o}^{+}$and $\mathrm{C}_{i}^{+}$). Orbits crossing $\partial^{-} \mathbf{P}$ either inside $\mathrm{C}_{i}^{-}$or outside $\mathrm{C}_{o}^{-}$leave $\mathrm{P}$ again by $\partial^{+} \mathrm{P}$, inside $\mathrm{C}_{i}^{+}$or outside $\mathrm{C}_{o}^{+}$. We give the vectorfield in $\mathrm{P}$ the "mirror property": that the involution $\mathrm{D} \times[-1,1] \rightarrow \mathrm{D} \times[-1,1]$ given by $(x, t) \rightarrow(x,-t)$ reverses the vectorfield. This insures that the Poincare map from $\partial^{-} \mathbf{P}$ to $\partial^{+} \mathbf{P}$ is the identity on $\mathrm{D}^{2}$. 
In our example, the cylinder joining $\sigma_{+}$to the closed source, and the disc formed by the corresponding cylinder for $\sigma_{-}$together with the disc inside $\mathrm{D} \times\{-1 / 2\}$ bounded by $\sigma_{-}$, are deleted from $\mathrm{X}$, so that they represent the behavior "at infinity". We note that the complement of a disc is homeomorphic to the complement of a point, while the complement of a cylinder is homeomorphic to the complement of a circle (e.g., note that $\mathrm{K}$ can be replaced by a tubular neighborhood, then use the isotopy extension theorem [2, p. 180]). From this point of view, the plug can be described more simply (Figure 6): we introduce a rest point $p=(0,0,1 / 2)$, and a closed orbit $\sigma=\mathrm{S}(1 / 2) \times\{1 / 2\}$, with the following features:

(i) the local stable and unstable sets of $p$ are solid cones with vertices at $p$,

(ii) $\sigma$ is of quasi-hyperbolic type: its stable manifold is a cylinder which coincides with the boundary of the solid cone $\mathrm{W}^{u}(p)$; its local unstable set is the solid formed by rotating about $\sigma$ a wedge with vertex on $\sigma$.

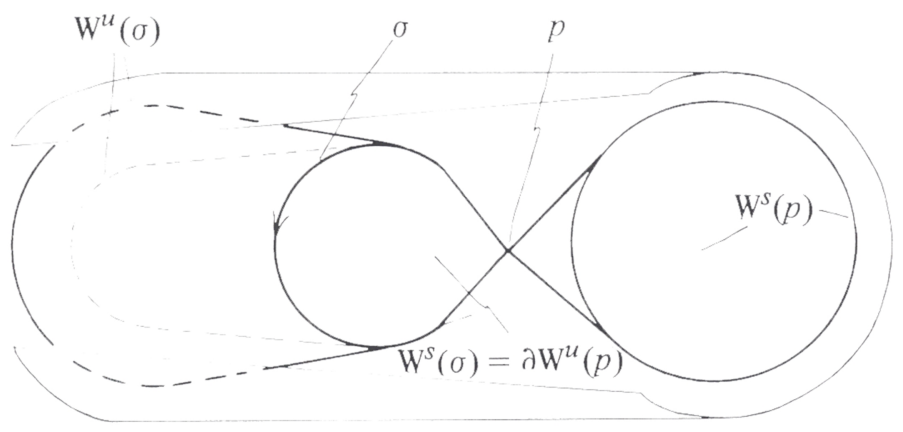

FigURE 6

We let $\mathrm{A}^{+}$be the annulus in which $\mathrm{W}^{u}(\sigma)$ meets ${ }^{+} \mathrm{P} ; \mathrm{C}_{o}^{+}$and $\mathrm{C}_{i}^{+}$denote its outer and inner boundary.

Although this second description is simpler, the first description makes it easier to see that by controlling the behavior of the flow near the degenerate rest point $p$ and the periodic orbit $\sigma$, we can insure the following properties as well:

(iii) For any arc meeting $\partial \mathrm{W}^{s}(p)$ transversally, the $e^{1}$-closure of its orbit includes $\partial \mathrm{W}^{u}(p)=\mathrm{W}^{s}(\sigma)$. (This is the $\lambda$-lemma for $\sigma_{-}$in the first description.)

(iv) The orbit of an arc meeting $\mathrm{W}^{s}(\sigma)$ transversally $\mathrm{e}^{\mathrm{l}}$-accumulates on both boundaries of $\mathrm{W}^{u}(\sigma)$ (the $\lambda$-lemma for $\sigma_{+}$).

(v) A point $(x,-1) \in \partial^{-} \mathrm{P}$ entering $\mathrm{P}$ outside $\mathrm{W}^{s}(p)$ leaves $\mathrm{P}$ via $(x,+1)$ $\in \partial^{+} \mathrm{P}$ (the mirror property).

Finally, the strong topology will insure a certain persistence to this picture 
under perturbation. Recall that the strong $\mathbb{C}^{1}$-topology replaces the constant $\varepsilon>0$ in the usual pointwise estimates with a function $\varepsilon(x)$, which is positive on the open manifold. This function can, however, vanish as rapidly as desired "at infinity". In particular, if $\phi$ is a flow defined on the closed

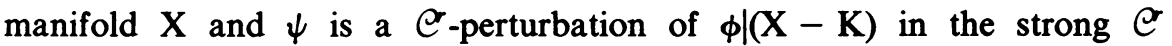
topology, then $\psi$ extends to a $C$ flow on $X$, which is a $\bigodot$ perturbation of $\phi$ (in the usual sense) on $\mathrm{X}$, and which moreover agrees with $\phi$, up to its $r$-jet, at points of $\mathrm{K}$. Combining this observation with the nondegeneracy conditions on $\sigma_{-}$and $\sigma_{+}$in our first description of the plug, we find

(vi) if $\psi$ is a strong- $C^{1}$ perturbation of $\phi$ on $\mathrm{P}-\{\sigma \cup p\}$, then properties (i)-(iv) above hold for $\psi$ as well, with the exception that $\mathrm{W}^{s}(\sigma)$ need no longer coincide with $\partial W^{u}(p)$. The various local invariant manifolds $\left(W^{s}(\sigma)\right.$, the two components of $\left.W^{u}(\sigma), \partial W^{u}(p), \partial W^{s}(p)\right)$ for $\psi$ are near those of $\phi$, and the closed orbit $\sigma$ and rest point $p$ for $\psi$ are identical to those for $\phi$.

The Pugh-Walker-Wilson example is constructed by inserting two plugs (of the type in the first description) into the basin of attraction of an attracting closed orbit, taking care to align them properly with respect to the flow. This step, which is the crux of the example, gives us a special case of Theorem 2:

Proposition 4. Let $\mathrm{X}$ be the open solid torus $\mathrm{D}^{2} \times \mathrm{S}^{2}$, and let $\mathrm{K}$ be the union of two unlinked, unknotted contractible circles in $\mathrm{X}$. Then $\mathbb{S}^{1}(\mathrm{X}-\mathrm{K})$ is not dense in $\mathfrak{W}^{1}(\mathrm{X}-\mathrm{K})$.

Proof. Using coordinates $z \in \mathrm{D}^{2}, \theta \in \mathrm{S}^{1}$, we begin with the flow

$$
\dot{z}=-z, \quad \dot{\theta}=1
$$

which has a single periodic, global attractor at $z=0$.

We will modify this flow with two plugs, $P_{1}$ and $P_{2}$, constructed according to the second description and aligning the annuli $A_{1}^{+} \subset \partial^{+} P_{1}$ and $A_{2}^{+}$ $\subset \partial^{+} \mathrm{P}_{2}$ carefully. (We use subscripts on $\mathrm{A}^{+}, p$, and $\sigma$ to indicate which plug they belong to, and denote the boundary circles of $\mathrm{A}_{j}^{+}$by $\mathrm{C}_{o}^{+}(j)$ and $\mathrm{C}_{i}^{+}(j)$.) Note that by the mirror property, we must have (for $j=1,2$ )

$$
\partial \mathrm{W}^{s}\left(p_{j}\right) \cap \partial^{-} \mathbf{P}_{j}=\mathrm{C}_{o}^{+}(j) \text {. }
$$

Before constructing the plugs, we specify the location of the flowboxes $\mathbf{P}_{1}$ and $\mathrm{P}_{2}$ and the distinguished circles $\mathrm{C}_{o}^{+}(j), \mathrm{C}_{i}^{+}(j)(j=1,2)$ (see Figure 7). Think of $P_{2}$ as placed directly "downstream" from $P_{1}$, so that points leaving $\partial^{+} P_{2}$ make most of a circuit around the torus (and are therefore contracted) before returning to $\partial^{-} P_{1}$. Our alignment hypotheses are:

1. The periodic attractor $z=0$ crosses $\partial^{+} \mathrm{P}_{j}$ interior to $\mathrm{A}_{j}^{+}$for $j=1$ and 2 .

2. The flow images of $\mathrm{C}_{o}^{+}(1)$ and $\mathrm{C}_{i}^{+}(1)$ both meet $\mathrm{C}_{o}^{-}(2)$ transversally.

3. The flow image of $\mathrm{A}_{j}^{+}$returns to $\mathrm{P}_{j}$ interior to $\mathrm{A}_{j}^{+}$.

4. When the flow image of $A_{2}^{+}$crosses $\partial^{-} P_{1}$, both boundary circles $C_{o}^{+}(2)$, $\mathrm{C}_{i}^{+}(2)$ intersect $\mathrm{C}_{o}^{+}(1)$ transversally. 


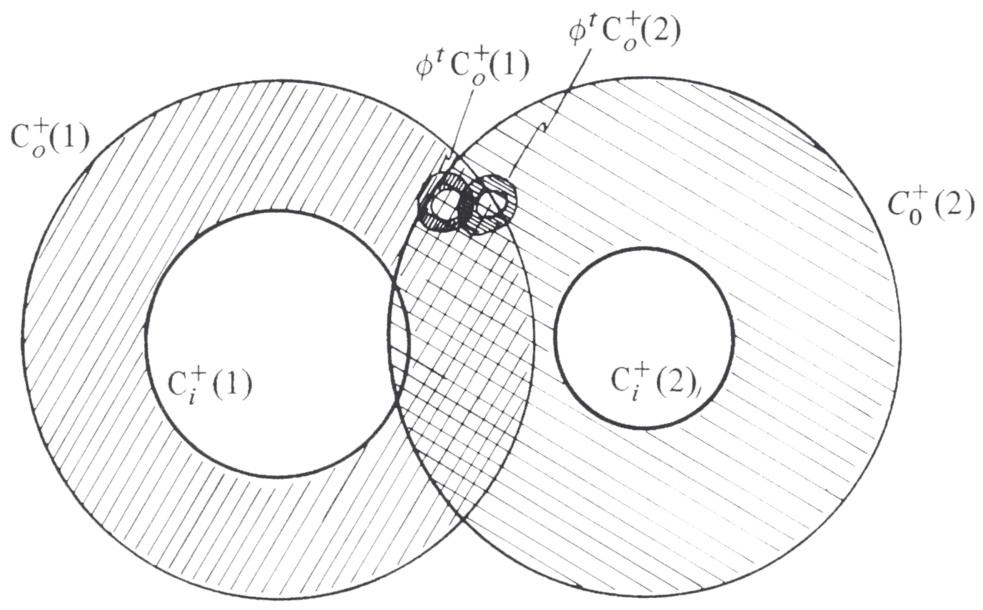

FIGURE 7

To prove the proposition, we need to establish two facts: that the plugged flow $\phi$ is completely unstable on the complement of $\mathrm{K}_{1}=\sigma_{1} \cup \sigma_{2} \cup p_{1} \cup p_{2}$ (equivalently, that on $\mathrm{X}, \Omega(\phi)=\mathrm{K}_{1}$ ), and that any strong-e $e^{1}$ perturbation of $\phi$ can be further $\mathrm{C}^{\mathrm{l}}$-perturbed to have other nonwandering points.

Claim $1 . \Omega(\phi)=\mathrm{K}_{1}$ in $\mathrm{X}$.

Proof. We note that, by the mirror property (v), any point leaving $\partial^{+} \mathrm{P}_{j}$ ( $j=1$ or 2 ) is either captured inside the other plug (that is, in $\mathrm{W}^{s}\left(p_{k}\right), k \neq j$ ) or returns to $\partial^{-} \mathrm{P}_{j}$ at the same point as it would reach by the unplugged flow. Thus, the only candidates for $\Omega$ are those in $\partial \mathrm{W}^{s}\left(p_{j}\right), \partial \mathrm{W}^{u}\left(p_{j}\right), \mathrm{W}^{s}\left(\sigma_{j}\right)$, or $\partial \mathrm{W}^{u}\left(\sigma_{j}\right)(j=1,2)$. However, a neighborhood of any one of these points leaves $\partial^{+} \mathrm{P}_{j}$ by an open set very near $\mathrm{A}_{j}^{+}$, and when this returns to $\partial^{-} \mathrm{P}_{j}$, it is captured in the interior of $\mathrm{A}_{j}^{-}$-that is, in $\mathrm{W}^{s}\left(p_{j}\right)$ (alignment hypothesis 3 ). This establishes the claim.

Claim 2. For a dense set of strong-e perturbations $\psi$ of $\phi$,

$$
\Omega\left(\psi \mid \mathrm{X}-\mathrm{K}_{1}\right) \neq \varnothing \text {. }
$$

Proof. By property (vi), we know that in both $\mathrm{P}_{1}$ and $\mathrm{P}_{2}$, the various invariant manifolds for $\psi$ will approximate those of $\phi$. In each plug, we consider the relation of $\mathrm{W}^{s}\left(\sigma_{j}\right)$ to $\partial \mathrm{W}^{u}\left(p_{j}\right)$. If they have a point of intersection, then it is clear that by a $e^{1}$ perturbation of $\psi$ supported on a small neighborhood of the intersection point (and disjoint from $\mathrm{K}_{1}$ ), we can introduce a point of transversal intersection. The limitation on the support insures that this is a strong-e perturbation on $X-K_{1}$. Thus, up to strong- $e^{1}$ perturbation, we can assume that in each plug, $\psi$ satisfies either

(a) $\mathrm{W}^{s}\left(\sigma_{j}\right) \cap \partial \mathrm{W}^{u}\left(p_{j}\right)=\varnothing$ 
or

(b) $\mathrm{W}^{s}\left(\sigma_{j}\right)$ has a point of transverse intersection with $\partial \mathrm{W}^{u}\left(p_{j}\right)$.

We note that in case (a), $\partial \mathrm{W}^{u}\left(p_{j}\right)$ leaves $\partial^{+} \mathrm{P}_{j}$ by a circle concentric with $\mathrm{C}_{o}^{+}(j)$, and either just outside $\mathrm{C}_{o}^{+}(j)$ or just inside $\mathrm{C}_{i}^{+}(j)$.

We wish to show that in either case, $\Omega\left(\psi \mid \mathrm{X}-\mathrm{K}_{1}\right) \neq \varnothing$. To this end, we pick an arbitrary point $x \in \partial \mathrm{W}^{s}\left(p_{1}\right)$, and trace its first prolongation

$$
\mathrm{J}^{+}(x)=\left\{y \mid \exists t_{i} \rightarrow+\infty, x_{i} \rightarrow x \text { such that } \psi\left(t_{i}, x_{i}\right) \rightarrow y\right\} .
$$

We will show that $\partial \mathrm{W}^{u}\left(p_{2}\right) \subset \mathrm{J}^{+}(x)$ for any $x \in \partial \mathrm{W}^{s}\left(p_{1}\right)$.

In fact, take a neighborhood $U$ of $p_{1}$ in $X$; by (iii) its orbit includes arcs nearly equal to any arc in $\partial W^{u}\left(p_{1}\right)$. But in case (a), this includes arbitrary approximations to every arc in one of the circles $\mathrm{C}_{o}^{+}(1)$ or $\mathrm{C}_{i}^{+}(1)$, while in case (b), (iv) implies that it includes approximations to all arcs in both circles. In either case, alignment hypothesis 2 implies that the orbit of $U$ includes arcs meeting $\mathrm{C}_{o}^{-}(2)$ and hence $\partial \mathrm{W}^{s}\left(p_{2}\right)$ transversally. But then (iii) implies that these arcs accumulate on all of $\partial W^{u}\left(p_{2}\right)$. Thus, any neighborhood $U$ of $x \in \partial \mathrm{W}^{s}\left(p_{1}\right)$ includes points whose orbits pass arbitrarily near any point of $\partial \mathrm{W}^{u}\left(p_{2}\right)$. That these points take increasingly long to approximate $\partial \mathrm{W}^{u}\left(p_{2}\right)$ follows from the fact that $x$ itself tends to $p_{1}$. Thus, $\partial \mathrm{W}^{u}\left(p_{2}\right) \subset \mathrm{J}^{+}(x)$.

Finally, we know that $\partial \mathrm{W}^{u}\left(p_{2}\right)$ has points of intersection with $\partial \mathrm{W}^{s}\left(p_{1}\right)$, by alignment hypothesis 4 and a discussion identical to the one following the statement of cases (a) and (b) above. Thus, if we pick $x$ in the containment above to be such a point of intersection, we obtain

$$
x \in \partial \mathrm{W}^{u}\left(p_{2}\right) \subset \mathrm{J}^{+}(x) .
$$

But this is equivalent to $x \in \Omega(\psi)$.

We have thus established Claim 2: that $\Omega\left(\psi \mid \mathbf{X}-\mathbf{K}_{1}\right) \neq \varnothing$. This would conclude the proof of Proposition 4, except that $K_{1}$ consists of two circles and two points, whereas the statement of the proposition requires $K=$ two circles. However, take a simple arc $\gamma_{1}$ joining $p_{1}$ to $p_{2}$ in $\mathrm{X}$, disjoint from $\sigma_{1}$ and intersecting $\sigma_{2}$ only in its endpoint, and then a simple arc $\gamma_{2}$ from $p_{2}$ to $\sigma_{2}$. We note that the arc $\gamma=\gamma_{1} \cup \gamma_{2}$ is so thin that it does not affect the arguments above: our intersection arguments can all be carried out away from $\gamma$. On the other hand, we can multiply the vectorfield by a nonnegative function vanishing precisely on $K=K_{1} \cup \gamma$, to make the vectorfield complete on $\mathrm{X}-\mathrm{K}$; note that $\mathrm{K}$ consists of the circle $\sigma_{1}$ and the circle $\sigma_{2}$ attached to the arc $\gamma$. Again, a tubular neighborhood-isotopy extension argument shows that $X-\left[\sigma_{1} \cup \sigma_{2} \cup \gamma\right]$ is homeomorphic to $X-\left[\sigma_{1} \cup \sigma_{2}\right]$, and this indeed proves Proposition 4. Q.E.D.

Proof of Theorem 2. To complete the proof of Theorem 2, we need to glue the example above into a flow on any 3-manifold $\mathrm{X}$. This is not hard:

Claim. Every 3-manifold X supports a flow $\phi$ whose nonwandering set 
consists of a contractible, unknotted attracting periodic orbit and, if $\mathrm{X}$ is closed, a single rest point.

Proof of Claim. This is essentially an adaptation of Theorem A in [7], or arguments in [8] and [11]. Every 3-manifold supports a nonsingular flow, which can be chosen to be completely unstable if $\mathrm{X}$ is open.

If $\mathrm{X}$ is closed, we wish to concentrate $\Omega(\phi)$ in a single rest point. There exists a finite, disjoint collection of flowboxes whose saturation is $\mathrm{X}[\mathbf{1 1}$, Theorem A]; by joining them with curves transverse to the flow and then thickening, one obtains a single flowbox whose saturation is $\mathrm{X}$. In this flowbox, introduce a degenerate rest point $p_{0}$ whose local stable and unstable sets are solid cones (like $p$ in our earlier construction), taking care to preserve the mirror property in the flowbox, and making sure that these cones meet the inset and outset in sets whose saturation is all of $\mathrm{X}$. Then every orbit in $\mathrm{X}$ emanates from the unstable cone and terminates in the stable cone, so $\Omega$ consists of the single rest point.

If $\mathrm{X}$ is open, we simply pick a flowbox and introduce a rest point $p_{0}$ as above; the mirror property and complete instability of the original flow insure that $\Omega$ is a single rest point.

Now, we introduce a periodic attractor inside the stable cone of $p_{0}$, by

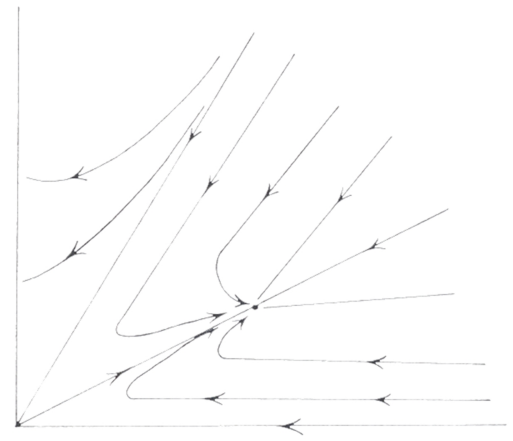

FigurE: 8a

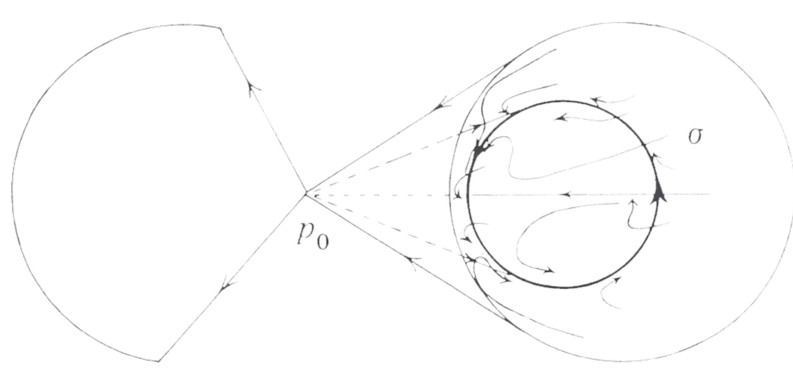

FIGURE 8 b 
rotating the flow in Figure $8 \mathrm{a}$ about the $x$-axis to obtain an attracting circle of fixedpoints, then adding a small rotation component near the circle. The flow is sketched in Figure $8 \mathrm{~b}$.

If $\mathrm{X}$ is closed, this establishes the claim; if $\mathrm{X}$ is open, we eliminate $p_{0}$ from $\mathrm{X}$ by joining $p_{0}$ to "infinity" via one of the orbits in the unstable cone, and deleting the whole resulting simple arc.

The claim now sets the stage for gluing in the example of Proposition 4. Take a small tubular neighborhood of the attractor, noting that the vectorfield on the bounding torus agrees with the restriction of the vectorfield of Proposition 4 to its boundary. Hence, we can replace the flow inside this solid torus by the flow of Proposition 4. Since nothing escapes from the torus, the resulting unperturbed flow $\phi$ on $\mathrm{X}$ has its whole nonwandering set concentrated in $p_{0}$ together with $\mathrm{K}$ (of Proposition 4), which is the union of two unlinked, unknotted contractible circles. As before, we can eliminate $p_{0}$ by joining it to one of the deleted circles by a simple arc, which we delete (or equivalently, include in $\mathrm{K}$ ).

The arguments of Proposition 4 then tell us that $\phi \in \mathfrak{W}^{1}(\mathbf{X}-\mathbf{K})$, but no strong- $\bigodot^{1}$ perturbation of $\phi$ can belong to $\mathbb{S}^{1}(\mathbf{X}-\mathbf{K})$.

Finally, to replace two circles with a figure eight, we join the two circles by an arc, and note that a tubular neighborhood of a figure eight is diffeomorphic to a tubular neighborhood of two circles joined by an arc. Q.E.D.

We close with some further comments concerning this last example.

First, we can replace $r=1$ with any $r \geqslant 1$ in Theorem 2, because on one hand, $C^{\mathrm{l}}$-persistent phenomena are also $\mathcal{C}$-persistent, and on the other hand, the perturbation achieving transverse intersection of $\mathrm{W}^{s}(\sigma)$ with $\partial \mathrm{W}^{u}(p)$ in case (b) can be made $C$ as well. We can equally replace $\operatorname{dim} X=3$ with $\operatorname{dim} \mathrm{X}=n \geqslant 3$ by taking the product of the flow in Proposition 4 (on $\mathrm{D}^{2} \times \mathrm{S}^{1}$ ) with the constant flow on $\mathrm{D}^{n-3}$, then gluing into a neighborhood of a periodic attractor as before. However if $X$ is closed, we need to pay attention to the Euler characteristic at the beginning of the proof of Theorem 2. But one can still create a flow with nonwandering set a single rest point, by taking the gradient of a Morse function, then deforming it so as to join the critical points into a single one of high index. Finally, this process must result in a stable set with interior (corresponding to the basin of attraction of a minimum of the Morse function), so that we can introduce the periodic attractor in this set, as before.

These observations can be summarized as

COROllaRY 2. For any $r \geqslant 1$ and any manifold $\mathrm{X}$ (open or closed) with $\operatorname{dim} \mathrm{X} \geqslant 3$, Let $\mathrm{K}$ denote the union of two circles bounding disjoint discs in $\mathrm{X}$. Then $\mathfrak{S S}^{r}(\mathrm{X}-\mathrm{K})$ is not dense in $\mathfrak{W}(\mathrm{X}-\mathrm{K})$. 
The manifolds described in Corollary 2 are a large class, but certainly not exhaustive. It is amusing to note that when $X=S^{3}$, Theorem 2 gives the result on the complement of a single circle in the solid torus, thus apparently extending Proposition 4. However, the boundary behavior on this torus does not permit the easy surgery in the proof of Theorem 2 . The conjecture on density remains open, then, for two general classes of open manifolds:

(i) open surfaces other than $R^{2}$,

(ii) punctured closed manifolds of the form $\mathrm{X}^{n}-\mathrm{K}, n \geqslant 3, \mathrm{X}$ closed, $\mathrm{K}$ a single circle, or a totally disconnected set.

For example, is $\mathbb{S}^{1}\left(\mathbf{R}^{3}\right)$ dense in $\mathfrak{B}^{1}\left(\mathbf{R}^{3}\right)$ ?

We note that our example also says nothing about $r=0$. The persistence statements in the proof of Proposition 4 no longer apply; indeed, one can, via strong- $\bigodot^{0}$ perturbations, "thicken" the cylinder $W^{s}(\sigma)$ into a set with nonempty interior, similar to $\mathrm{W}^{u}(\sigma)$. Then, by making sure $\partial \mathrm{W}^{u}(p)$ is interior to $\mathrm{W}^{s}(\sigma)$, one obtains a strong- $\mathrm{e}^{0}$ perturbation of $\phi$ with empty Auslander recurrent set ([4]) and hence in $\mathbb{S}^{\circ}(\mathrm{X}-\mathrm{K})$.

As a final remark, we pose a question related to both parts of this paper. We have seen in Theorem 1 that, when $\mathfrak{B}^{r}(\mathrm{M})$ has a nonempty frontier (relative to $\mathfrak{R}^{r}(\mathbf{M})$ ), then this frontier contains both explosive flows in $\mathfrak{B}^{r}(\mathbf{M})$ and "implosive" flows with $\Omega \neq \varnothing$. One might ask which kind of flow is more prevalent in the boundary-especially in the boundary of (sr (M). The examples in Theorem 1 involving quasi-hyperbolic periodic orbits can be shown, using standard compact theory (see, e.g., [3]) to be ${ }^{1}$ persistent as bifurcation points. On the other hand, two-dimensional examples [6] seem to indicate that explosive flows have codimension 2 or more. Thus, we ask a

Question. Let $\mathfrak{X}_{u}:[0,1] \rightarrow \mathfrak{R}^{1}(\mathrm{M})$ be a strong arc with $\mathfrak{X}_{u} \in \mathbb{B}^{1}(\mathbf{M})$ for $u<1 / 2$ and such that $\mathfrak{X}_{u}$ is interior to $\mathfrak{R}^{1}(\mathrm{M})-\mathfrak{B}^{1}(\mathrm{M})$ for $u>1 / 2$. Is it generically true (in the strong- $\bigodot^{1}$ topology on strong arcs) that $\Omega\left(\mathscr{X}_{1 / 2}\right) \neq \varnothing$ ?

If we follow $\mathfrak{X}_{u}$ for decreasing $u$, then the periodic points in $\Omega\left(\mathscr{X}_{u}\right)$ may vanish by means of a saddle-node type of bifurcation, but it is also possible that they vanish to infinity, in some sense. It is the latter type of bifurcation which must be understood, to answer this question.

NOTE ADDED IN PROOF. For punctured closed surfaces $M=X^{2}-K, X$ closed, $\mathrm{K}$ a finite set of points, the phenomenon exhibited by our three-dimensional examples in $\$ 2$ does not occur, and the question at the end of this paper has an affirmative answer. Details will appear in the author's forthcoming paper, Recurrent structure of completely unstable flows on surfaces of finite genus.

\section{REFERENCES}

1. M. W. Hirsch, On embedding differentiable manifolds in Euclidean space, Ann. of Math. (2) 73 (1961), 566-571.

2. ___ Differential topology, Springer-Verlag, Berlin and New York, 1976. 
3. S. Newhouse and J. Palis, Bifurcations of Morse-Smale dynamical systems, Dynamical Systems (ed. M. Peixoto), Academic Press, New York, 1973, pp. 303-366.

4. Z. Nitecki, Explosions in completely unstable flows. I. Preventing explasions, Trans. Amer. Math. Soc. 245 (1978), 43-61.

5. Explosions in completely unstable flows. II. Some examples, Trans. Amer. Math. Soc. 245 (1978), 63-88.

6. Bifurcation from completely unstable flows on the cylinder, Ann. New York Acad. Sci. 316 (1979), 86-109.

7. P. Percell, Presentations of 3-manifolds arising from vector fields, Trans. Amer. Math. Soc. 221 (1976), 361-377.

8. P. Percell and F. W. Wilson, Jr., Plugging flows, Trans. Amer. Math. Soc. 233 (1977), 93-103.

9. C. C. Pugh, R. Walker and F. W. Wilson, Jr., On Morse-Smale approximations-a counterexample, J. Differential Equations 23 (1977), 173-182.

10. F. Takens and W. White, Vectorfields with no nonwandering points, Amer. J. Math. 98 (1976), 415-425.

11. F. W. Wilson, Jr., On the minimal sets of non-singular vector fields, Ann. of Math. (2) 84 (1966), 529-536.

Department of Mathematics, TUfts University, Medford, Massachusetts 02155 